\title{
Extended VCS Stark broadening tables for hydrogen - Lyman to Brackett series
}

\author{
M. Lemke ${ }^{1, \star}$ \\ Institute of Astronomy, Madingley Road, Cambridge CB3 0HA, UK \\ e-mail: michael@astro.as.utexas.edu
}

Received October 4, 1995; accepted July 30, 1996

\begin{abstract}
We announce the availability of Stark broadening tables for the Lyman, Balmer, Paschen, and Brackett series of hydrogen, computed with the VCS theory (Vidal et al. 1973). The tables cover the series up to $n=22$ and an extended grid of $n_{\mathrm{e}}$ and $T$, with $T$ running as high as $160000 \mathrm{~K}$, suitable for central stars of Planetary Nebulae and similar ${ }^{1}$.
\end{abstract}

Key words: atomic data — line: profiles

\section{Introduction}

Hydrogen lines provide an important diagnostic tool for determining fundamental stellar parameters like effective temperature or gravity. This diagnostic means is mainly facilitated by the fact that the line broadening is almost completely determined by Linear Stark effect. A very successful theoretical description of this crucial broadening mechanism has been developed by Vidal et al. (1973, and references therein) and their tables have been in wide use since then. Unfortunately, the tables cover only the first four lines of the Balmer and Lyman series, which is rather limiting in lieu of the spectral coverage obtainable with current detectors and space experiments. Despite more elaborate broadening theories having been developed only recently has a set of tables been computed that is applicable to stellar envelopes (Stehlé 1994, 1996) and that extends to lines other than $\operatorname{Ly} \alpha-\operatorname{Ly} \delta$ and $\mathrm{H} \alpha-\mathrm{H} \delta$. These new tabulations are based on the Model Microfield Method (or MMM, Frisch \& Brissaud 1971), which should be more accurate than VCS for taking temporal statistics between the perturbers into account. Regrettably, with

Send offprint requests to: M. Lemke, Bamberg address

* Present address: Dr.-Remeis-Sternwarte, Sternwartstr. 7, D-96049 Bamberg, Germany.

1 The tables are available at the CDS via anonymous ftp 130.79.128.5 or http://cdsweb.u-strasbg.fr/Abstract.html a lowest temperature of $10000 \mathrm{~K}$, and only two decades in electron density, the size of the Stehlé tables seriously restricts their application. Fortunately, for many astrophysical purposes where Doppler broadening is significant the differences between VCS and MMM appear to be extremely small. Napiwotzki \& Rauch (1994), who used similar Stark broadening calculations by Schöning, could not find significant differences between spectra computed with either method for a wide range of stellar parameters $(T \geq 20000 \mathrm{~K})$. The Schöning calculations are not strict MMM calculations, however, but only a simplified approximation to MMM. The differences to Stehlé seem to be small (cf. Stehlé Fig. 2 and Schöning Fig. 1) and in the sense which should increase the deviations from VCS.

Having had a pressing need to analyze Paschen lines (Lemke \& Venn 1996) and realizing that VCS published their computer code, we felt that after 25 years the VCS tables are still useful and should be extended to higher lines. No intention was made, however, to improve on or modify the VCS description as it was laid out in VCS 1973.

In the present paper we announce the availability of VCS tables for the Lyman, Balmer, Paschen, and Brackett series up to $n=22$, computed with the original program.

\section{Method}

We took the computer code from Vidal et al. (1971) and applied the modifications from Vidal et al. (1973) for the $B$ constant. We reworked the code slightly (essentially array dimensions) to allow for higher quantum numbers. No modifications were applied that change any of the physics or the mathematical descriptions. We therefore refer the reader to the original work (in particular VCS 1971) for details on validity and limitations of the VCS method and its implementation. For the present paper we tried to stay as close as possible to the original work. A few points, however, shall be raised here. 


\subsection{Limitations}

Both VCS and MMM description have the limitation of not taking into account quasi molecules that form with the perturbers during the collision with the hydrogen atoms. These quasi molecules produce so-called satellite lines at large distances from the line center. The collision of neutral hydrogen with other neutral hydrogen atoms, for example, leads to the temporary formation of an $\mathrm{H}_{2}$ molecule, which is responsible for the " $1600 \AA$ feature", a conspicuous broad depression in the UV flux of white dwarfs and $\lambda$ Boo stars (Koester et al. 1985; Nelan \& Wegner 1985; Holweger et al. 1994). For the feature to be visible it is necessary that Ly $\alpha$ is strong enough at about $1600 \AA$ (white dwarfs, A stars) and that it is not obscured by metal lines (low-metallicity effect of $\lambda$ Boo stars). Similar perturbations with protons produce sharper features, of which there is a very strong one near $1400 \AA$. That "line" is very visible in white dwarfs but like the $1600 \AA$ feature can be masked by metal lines in A-type stars (cf. Fig. 4 in Holweger et al.) although to a lesser extent. This suggests that the importance of quasi molecules is restricted to high gravity/low metallicity objects and apparently also to Ly $\alpha$. Even though a VCS type profile is completely wrong under such conditions, for most objects satellite features will not be important and MMM or VCS profiles will adequately reproduce the line shape.

Our tables reach to lower electron densities (see Sect. 3) than the original work. For the lowest values of $n_{\mathrm{e}}$ and $T$ influences of the neglected fine structure are to be expected. For astrophysical purposes, however, this should not pose a problem as Doppler broadening will dominate; but care must be taken if the pure Stark profiles are investigated. For the higher lines one should be aware that those lines are no longer isolated. Therefore, quenching effects, which have not been taken into account, will play a role. For most practical cases it is good enough to simply add the contributions of neighboring lines (cf. VCS 1973). Specifically for white dwarfs Bergeron (1993) devised an approximate correction for quenching effects to be used with existing VCS tables.

\subsection{Application}

Following VCS (1971), perturbations of the lower state sublevels by the electrons (lower state interaction) were only included for the first three lines in a series (i.e., $\alpha$ to $\gamma$ ); for intermediate series members the Stark effect for only the static ions on the lower level was taken into account. For the highest lines, i.e., for $n>11,10,13$ for the Balmer, Paschen, Brackett series, respectively, the lower state was completely ignored, i.e., we did not compute those profiles but took the Lyman profile with the same upper $n$ instead. According to VCS (1971), this introduces an error less than that due to the underlying physical as- sumptions. In Fig. 1 we show a case for $n=10$. The maximum error in this case is $7 \%$ for the Paschen line, which confirms the results by VCS. The Lyman profiles were scaled to the higher series as

$$
S(\Delta \alpha)=S_{\mathrm{Ly}}\left(\Delta \alpha_{\mathrm{Ly}}\left(\frac{\lambda_{0}}{\lambda_{0, \mathrm{Ly}}}\right)^{2}\right) /\left(\frac{\lambda_{0}}{\lambda_{0, \mathrm{Ly}}}\right)^{2}
$$

where $S$ is the normalized Stark profile, $\Delta \alpha$ the distance from the line center in units of the normal field strength $F_{0}, \lambda_{0}$ the central vacuum wavelength of the respective line, and the index Ly denotes the corresponding quantity for the Lyman line with the same upper $n$.

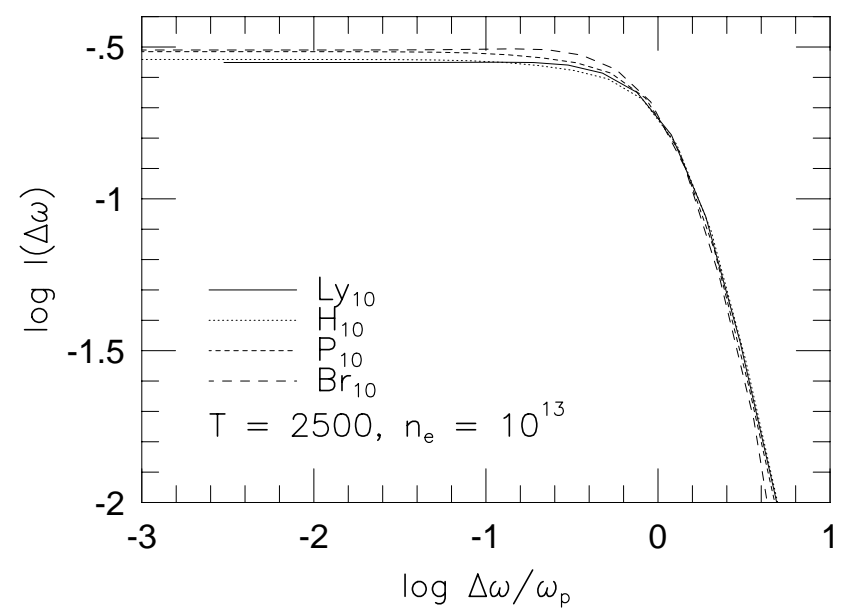

Fig. 1. Stark profiles for Lyman through Brackett for $n=10$ as function of the plasma frequency. Profiles normalized for $\int_{0}^{\infty} I(\Delta \omega) \mathrm{d} \Delta \omega / \omega_{\mathrm{p}}=1$

The code turned out to be numerically somewhat unstable, in particular at the transition region to the asymptotic wing. Tentatively, we replaced several routines with counterparts from the commercial NAG library but while this made the code more stable it hardly changed any results. Eventually, we only replaced the Weddle integration routine (a variant of Simpson's Rule) with a potentially more accurate Romberg-type integration (a variable stepsize extrapolation method). Again, this is not significant for the results but only removes a few "wiggles" in the transition region. These experiments give confidence in the results and that the code is working properly. With these modifications we could easily reproduce the pure Stark profiles of the 1973 VCS tables within a few tenths of a percent.

Eventually, the profiles were convolved with Doppler profiles of the corresponding temperature. Note that the convolution destroys the scalability of the higher Lyman profiles as the Doppler width scales differently $\left(\propto \lambda / \lambda_{0}\right)$ than the Stark profile $\left(\propto\left(\lambda / \lambda_{0}\right)^{2}\right)$. The convolution was done by solving the convolution integral directly with an 
adaptive step size integration routine (q1dax in double precision) from the publically available CMLIB/Q1DA package. Unlike the case of the pure Stark profiles, our Doppler-broadened profiles are slightly different than the original VCS (1973) tables for the regions where our tables overlap with Vidal et al.'s. The reason for this is not easy to assess as Vidal et al. do not specify any details of their broadening procedure. Part of the difference is due to how close to the line center a profile has been computed. VCS sometimes go closer than we do. The maximum distance from the line center $\left(\Delta \alpha^{\max }\right)$ has been chosen such that the Doppler broadened profile can safely be extrapolated with a $\Delta \alpha^{-5 / 2}$ law, should that be necessary. Of course, the caveat of neglected satellite features (Sect. 2.1) applies.

The required computer time is still too high for direct inclusion of the code into, e.g., a line analysis program. While a single point of a Balmer line profile can be computed on a $70 \mathrm{MHz}$ Sun SparcStation within $0.1-20 \mathrm{~s}$, depending on the distance to the line center, the computer time grows enormously for the higher series; the whole tables for $\operatorname{Br} \alpha$ took about 2 weeks of $\mathrm{CPU}$ time. The interpolation in precomputed tables is therefore still required, even for the Lyman series, which is the quickest to compute.

\subsection{Comparison with $M M M$}

As outlined in the introduction part of the tables presented here are also covered by the MMM tables of Stehlé. As a further guide for the potential user of the tables and to further investigate the points made in the introduction we computed sample spectra of $\mathrm{H} \beta$ for a model atmosphere of $T_{\text {eff }}=20500 \mathrm{~K}$ and $\log g=3.75$ with solar composition. Three profiles were computed: one with the tables from Stehlé, one with our tables and a third one with our tables but where we extracted only a subset from our tables which corresponds exactly to the size of the Stehle tables. The resulting profiles are displayed in Fig. 2. To assess the differences between VCS and MMM the profiles computed with tables of the same size have to be compared. As can be seen from the dashed and solid lines in Fig. 2 the differences are indeed very small. The effects of the small table size, on the other hand, are significantly bigger as the dashed and dotted lines demonstrate. Similar results were obtained for a $10000 \mathrm{~K} / \log g=4.0$ model, representative for an A star.

\section{Description of tables}

The tables are available only electronically from the Centre de Données astronomique de Strasbourg (CDS $)^{2}$. They cover the Lyman, Balmer, Paschen, and Brackett series up to $n=22$ for a slightly extended grid of $T$ and

2 They may temporarily also be available from http://www.sternwarte.uni-erlangen.de/ftp/michael/vcs/

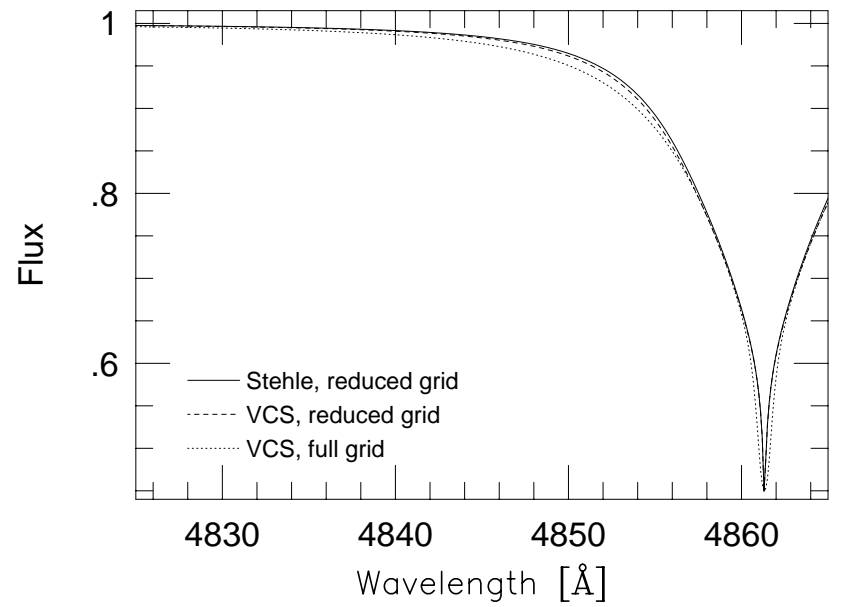

Fig. 2. $\mathrm{H} \beta$ profiles for $T_{\text {eff }}=20500 \mathrm{~K}, \log g=3.75$. Shown are profiles computed with our tables (dotted line) and with the tables from Stehlé (solid line). Also shown is a profile computed with our tables but with the parameter grid set identical to that of Stehlé (dashed line)

$n_{\mathrm{e}}$ than the 1973 tables. The tables contain the logarithm of the normalized Stark profile $S(\Delta \alpha)$ with

$\Delta \alpha=\Delta \lambda / F_{0}$,

where the distance $\Delta \lambda$ from the line center is in Ångströms and the normal field strength $F_{0}$ (in esu) is given as

$F_{0}=1.2510^{-9} n_{\mathrm{e}}^{2 / 3}$.

Normalization was such that

$\int_{0}^{\infty} S(\Delta \alpha) \mathrm{d} \Delta \alpha=1$

Unlike the original tables the grid is rectangular for easy interpolation and covers the range $2500 \leq T \leq 160000 \mathrm{~K}$; $10^{10} \leq n_{\mathrm{e}} \leq 10^{18} \mathrm{~cm}^{-3}$; and $10^{-3}\left(10^{-5}\right.$ for some higher lines) $\leq \Delta \alpha \leq 10^{3}$, all in the same steps as the original tables: $\Delta \log T=\log 2, \Delta \log n_{\mathrm{e}}=0.5, \Delta \log \Delta \alpha=0.2$. Note that the scaling of the Lyman lines results in a slightly different $\Delta \alpha^{\min }$ for the higher lines.

The code was not able to compute profiles for the higher lines of a series for high $n_{\mathrm{e}}$ and low $T$. This simply reflects the fact that the upper level does no longer exist for such high densities. However, for practical applications it is much easier to deal with a rectangular table instead of having the number of points vary with $n_{\mathrm{e}}$ and $T$. To ease the use of the tables we simply copied the last correctly produced profile to the lower temperature and indicate this fact with a flag (see Table 3). This allows easy interpolation and reading of the table with a single Fortran read statement. But we caution the reader that such profiles are not physically meaningful.

The pure Stark profiles and the broadened profiles are given in separate files. The file layout is given in Table 1 
and a minimal Fortran illustration for how to read it is given in Table 2.

Note added in proofs: After the paper had been accepted for publication it was realized that the unbroadened profiles were computed not close enough to the line center. As a result, the cores and small parts of the wings of the Doppler-broadened profiles were too shallow. This was most serious for Ly $\alpha$. We have therefore extended the affected profiles further down towards the line center. In correction to Sect. 3, Ly $\alpha-\operatorname{Ly} 9, \mathrm{H} \alpha-\mathrm{H} 11$, and $\mathrm{Pa} \alpha-\mathrm{Pa} \gamma$ are all computed down to $\Delta \alpha=10^{-8}$. This removes the remaining differences between our tables and VCS'. The Doppler-broadened profiles are tabulated only with as many points as are neccessary to recover the line center. Consequently, the smallest tabulated $\Delta \alpha$ varies from line to line; the first point is also valid for all smaller values of $\Delta \alpha$. A table with $\log \Delta \alpha^{\text {min }}$ for all lines can be found on the ftp servers. The information is of course contained in the actual profile tables as defined in Table 1.

Acknowledgements. I thank Tony Lynas-Gray for bringing to my attention the existence of the code and for having it installed and put at my disposal. I am also thankful to Dieter Engelhardt, Ralf Napiwotzki, and Thomas Rauch for useful discussions. The computations were carried out in parallel on several Starlink-provided SparcStations at the Institute of Astronomy, Cambridge. Financial support from the Leverhulme foundation under grant F627C is thankfully acknowledged.

\section{References}

Bergeron P., 1993, White Dwarfs: Advances in Observation and Theory. In: Barstow M.A. (ed.). Dordrecht/Boston/London Kluver Academic Press, p. 267

Frisch U., Brissaud A., 1971, JQSRT 11, 1753

Holweger H., Koester D., Allard N.F., 1994, A\&A 290, L21

Koester D., Weidemann V., Zeidler-K.T. E.-M., Vauclair G., 1985, A\&A 142, L5

Lemke M., Venn K.A., 1996, A\&A 309, 558

Napiwotzki R., Rauch T., 1994, A\&A 285, 603

Nelan E.P., Wegner G., 1985, ApJ 289, L31

Schöning T., 1994, A\&A 282, 994

Stehlé C., 1994, A\&AS 104, 509

Stehlé C., 1996, in: Vienna International Workshop on Model Atmospheres and Spectrum syntheses for mid-B to mid-G stars, at or close to the Main Sequence, July 6 to July 11 1995 (to be published in Conf. Ser. ASP)

Vidal C.R., Cooper J., Smith E.W., 1971, NBS Monograph 120

Vidal C.R., Cooper J., Smith E.W., 1973, ApJS 25, 37 
Table 1. File layout. The "goodness flag" is defined as follows: 0 : profile ok; -1 : copied from next higher $T$, -2 : copied from 2nd next higher $T$ etc.; -10: copied from next lower $n_{\mathrm{e}},-20$ : copied from 2 nd next lower $n_{\mathrm{e}}$ etc; -23 : copied from 2 nd next lower $n_{\mathrm{e}}$, then copied down three $T$ steps

$N$ :

For each line ( $N$ many):

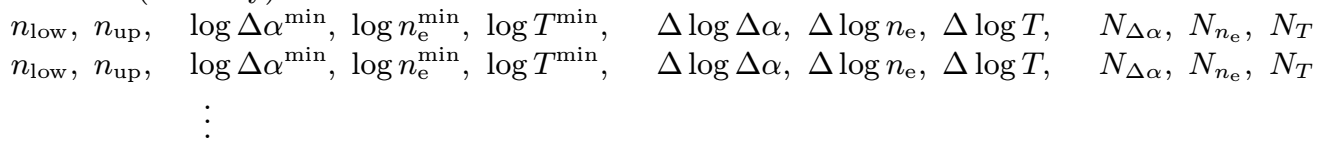

Profiles follow for each hydrogen line in sequence as specified above ( $N$ many): $\mathrm{nl}=\mathrm{ll} \mathrm{nu}=\mathrm{uu}$ $n_{\text {low }}$ and $n_{\text {up }}$ in I 2.2 format (consistency check)

For each $n_{\mathrm{e}}\left(N_{n_{\mathrm{e}}}\right.$ many): goodness flag, $N_{\Delta \alpha}$ profile points goodness flag, $N_{\Delta \alpha}$ profile points

lowest $T$, first $n_{\mathrm{e}}$ next $T$, first $n_{\mathrm{e}}$

goodness flag, $N_{\Delta \alpha}$ profile points goodness flag, $N_{\Delta \alpha}$ profile points

lowest $T$, next $n_{\mathrm{e}}$ next $T$, next $n_{\mathrm{e}}$ number of hydrogen lines in file

Next hydrogen line...

\section{$\mathrm{nl}=\mathrm{ll} \mathrm{nu}=\mathrm{uu}$}

For each $n_{\mathrm{e}}\left(N_{n_{\mathrm{e}}}\right.$ many): goodness flag, $N_{\Delta \alpha}$ profile points goodness flag, $N_{\Delta \alpha}$ profile points

$$
\text { : }
$$

goodness flag, $N_{\Delta \alpha}$ profile points goodness flag, $N_{\Delta \alpha}$ profile points $n_{\text {low }}$ and $n_{\text {up }}$ in I2.2 format (consistency check)

lowest $T$, first $n_{\mathrm{e}}$ next $T$, first $n_{\mathrm{e}}$

lowest $T$, next $n_{\mathrm{e}}$ next $T$, next $n_{\mathrm{e}}$

Example:

\begin{tabular}{|c|c|c|c|c|c|c|c|c|}
\hline \multicolumn{9}{|l|}{2} \\
\hline 12 & -3.77746 & \multicolumn{2}{|c|}{610.03 .39794} & \multicolumn{2}{|c|}{0.50 .301030} & \multicolumn{3}{|l|}{823} \\
\hline 213 & -3.78023 & 310.03. & 39794 & 20.50 .3 & 01030 & 823 & & \\
\hline \multicolumn{9}{|c|}{$\mathrm{nl}=02 \mathrm{nu}=12$} \\
\hline 0.0000 & -0.0369 & -0.0369 & -0.0369 & -0.0369 & -0.0370 & -0.0373 & -0.0380 & -0.0397 \\
\hline 0.0000 & 0.0234 & 0.0234 & 0.0233 & 0.0232 & 0.0230 & 0.0201 & 0.0189 & 0.0159 \\
\hline 0.0000 & 0.0981 & 0.0981 & 0.0980 & 0.0979 & 0.0975 & 0.0966 & 0.0943 & 0.0885 \\
\hline 0.0000 & 0.08 & 0.0981 & 0.0980 & 0.0979 & 0.0975 & 0.0966 & 0.0943 & 885 \\
\hline 0.0000 & -0.0521 & -0.0521 & -0.0521 & -0.0521 & -0.0522 & -0.0524 & -0.0530 & -0.0544 \\
\hline 0.0000 & 0.0053 & 0.0053 & 0.0053 & 0.0053 & 0.0051 & 0.0047 & 0.0037 & 0.0011 \\
\hline \multicolumn{9}{|c|}{$\mathrm{nl}=02 \mathrm{nu}=13$} \\
\hline 0.0000 & -0.2974 & -0.2974 & -0.2974 & -0.2973 & -0.2973 & -0.2972 & -0.2971 & -0.2966 \\
\hline 0.0000 & -0.3224 & -0.3224 & -0.3224 & -0.3224 & -0.3224 & -0.3222 & -0.3219 & -0.3211 \\
\hline 0.0000 & -0.3582 & -0.3582 & -0.3582 & -0.3582 & -0.3581 & -0.3579 & -0.3573 & -0.3560 \\
\hline 0.0000 & -0.2854 & -0.2854 & -0.2854 & -0.2854 & -0.2854 & -0.2853 & -0.2853 & -0.2851 \\
\hline 0.0000 & -0.2982 & -0.2982 & -0.2982 & -0.2982 & -0.2982 & -0.2982 & -0.2980 & -0.2977 \\
\hline 0.0000 & -0.3204 & -0.3204 & -0.3204 & -0.3203 & -0.3203 & -0.3202 & -0.3199 & -0.3192 \\
\hline
\end{tabular}


Table 2. Fortran illustration for reading table

\section{program vcs_list \\ implicit none}

integer pmne, pmp, pmt, mline

$>$

$>$

$>$

$>$

\begin{tabular}{|c|c|}
\hline real & svcs $($ pmt, pmne, 0:pr \\
\hline real & $\begin{array}{l}\text { log_alpha0(mline), } \\
\text { log_ne } 0 \text { (mline), } \\
\text { log_t0(mline) }\end{array}$ \\
\hline real & $\begin{array}{l}\text { log_alpha_inc(mline), } \\
\text { log_t_inc(mline), } \\
\text { log_ne_inc(mline) }\end{array}$ \\
\hline
\end{tabular}

integer $\mathrm{nl}(\mathrm{mline}), \mathrm{nu}(\mathrm{mline})$,

$>$

integer i, ip, ine, it, line, nline, nnl, nnu

$\mathrm{C} * * *$

$\mathrm{C} * * *$

$\mathrm{C} * * *$

Read in VCS arrays

$\operatorname{read}(5, *)$ nline

do $\mathrm{i}=1$, nline

$\operatorname{read}(5, *) \mathrm{nl}(\mathrm{i}), \mathrm{nu}(\mathrm{i})$,
$>\quad$ log_alpha0(i), log_ne0(i), log_t0(i),
$>\quad$ log_alpha_inc(i), log_ne_inc(i), log_t_inc(i),
$>\quad \mathrm{mp}(\mathrm{i}), \mathrm{mne}(\mathrm{i}), \mathrm{mt}(\mathrm{i})$

end do

$$
\begin{aligned}
& ! N_{n_{\mathrm{e}}} \quad\left(\max \# \text { of } n_{\mathrm{e}}\right) \\
& ! N_{T} \quad(\max \# \text { of } T) \\
& ! N_{\Delta \alpha} \quad(\max \# \text { of profile points }) \\
& ! N \quad(\max \# \text { of } \mathrm{H} \text { lines })
\end{aligned}
$$

\section{! VCS profiles}

$! \log \Delta \alpha^{\min }$

$! \log n_{\mathrm{e}}^{\min }$

$! \log T^{\min }$

! $\Delta \log \Delta \alpha$

! $\Delta \log n_{\mathrm{e}}$

! $\Delta \log T$

do line $=1$, nline

$\left.\operatorname{read}(5, '(3 x, i 2,4 x, i 2))^{\prime}\right) n n l, n n u$

$>$

! check against mline for array size

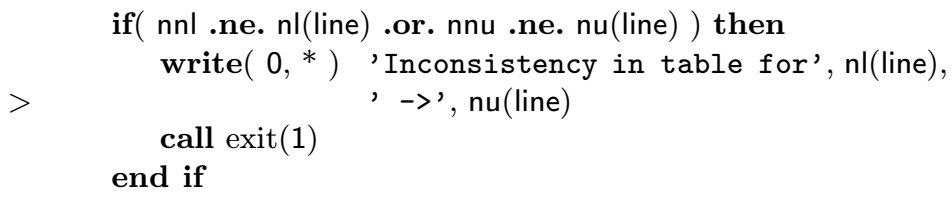

if $(\mathrm{nnl}$.ne. $\mathrm{nl}($ line $)$.or. nnu .ne. nu(line) ) then

write $\left(0,{ }^{*}\right)$ 'Inconsistency in table for', $\mathrm{nl}$ (line),

call exit(1)

end if

$\operatorname{read}(5, *)(((\operatorname{svcs}($ it, ine,ip,line $)$, ip $=0, \mathrm{mp}($ line $))$,

$>\quad$ it $=1, \mathrm{mt}($ line $))$,

$>\quad$ ine $=1$, mne(line $))$

end do

$\mathrm{C} * * *$

$\mathrm{C} * * *$

$\mathrm{C} * * *$

$\mathrm{C} * * * \quad \operatorname{svcs}(\ldots, \ldots, 0, \ldots)$ is goodness flag (cf. Table 1).

$\mathrm{C} * * *$

end 
Table 3. Overview of explicitly computed tables and goodness flag. The higher profiles not displayed here are scaled Lyman profiles (see text). Shown is the absolute value of the goodness flag as defined in Table 1. The abbreviated temperatures stand for $2500,5000,10000,20000,40000,80000,160000 \mathrm{~K}$, respectively

\begin{tabular}{|c|c|c|c|c|c|c|c|c|c|}
\hline $\log n_{\mathrm{e}}$ & $\operatorname{Ly} \alpha$ & $\operatorname{Ly} \beta$ & Ly $\gamma$ & Ly $\delta$ & $\mathrm{Ly} \epsilon$ & Ly 7 & Ly 8 & Ly 9 & Ly 10 \\
\hline 10 & O OOOOOO & OOOOOOO & O OOOOOO & O O O O O O O & O O O O O O O & O O O O O O O & O OOOOOO & O O O O O O O & O O O O O O O \\
\hline 10.5 & OOOOOOO & O O OOO O O & O O O O O O O & O O O O O O O & O O O O O O O & O O O O O O O & O O O O O O O & O O O O O O O & O OO OOO O \\
\hline 11 & O O OOO O O & O O OOO O O & OOOOOOO & O O O O O O O & O O O O O O O & O O OO OO O & O O OOOOO & O O OOOOO & O OOOOOO \\
\hline 11.5 & O O OOOOO & O O OOO O O & O O O O O O O & O O O O O O O & O O O O O O O & O O O O O O O & O O OOOOO & O O O O O O O & O O O O O O O \\
\hline 12 & O O OOOOO & O O OO OO O & O OO OO O O & O O O O O O O & O O OOO O O & O OOOOOO & OOOOOOO & O OOOOOO & O OO OOO O \\
\hline 12.5 & O O O O O O O & O O O O O O O & O O O O O O O & O O O O O O O & O O O O O O O & O O O O O O O & O O O O O O O & O O O O O O O & O O O O O O \\
\hline 13 & O O OOOOO & O OOOOO O & O OO OO O O & O OOOOOO & O O O O O O O & O O O O O O O & O OOOOO O & O O OOO O O & OOO OOO O \\
\hline 13.5 & O O O O O O O & O O OO OO O & O O O O O O O & O O O O O O O & O O O O O O O & O O O O O O O & O O O O O O O & O O O O O O O & O O O O O O O \\
\hline 14.5 & O O OO OO O & O O OO OO O & O O O O O O O & O O O O O O O & O O O O O O O & O O O O O O O & O OOOOOO & O O O O O O O & O O O O O O \\
\hline 15 & OOOOOOO & OOOOOOO & O OOOOOO & OOOOOOO & O O O O O O O & O O O O O O O & 0000 & o o o & O O O \\
\hline 15.5 & O O OOOOO & OOOOOOO & OOOOOOO & OOOOOOO & O OOOOOO & O O O O O O O & o o o & 1 o c & O O O \\
\hline 16 & OOOOOOO & OOOOOOO & O OO OO O O & O OOOOOO & O O OOO O O & 100 о о о о & 000 & O O O o & o o o \\
\hline 16.5 & O OOOOOO & OOOOOOO & OOOOOOO & 1 о о о о о о & 1 o о о о о о & 21 о о о о о & 21 о о о о о & 21 о о о о о & 321 о о о о \\
\hline 17 & O O O O O O O & O O OO OO O & 1 о о о о о о & 1 о о о о о о & 21 о о о о о & 21 о о о о о & 321 o o o o & 321 o o o o & 321 о о о о \\
\hline 17.5 & O O O O O O O & O O O O O O O & 1 o o o o o o & 21 o o o o o & 321 o o o o & 321 о о о о & 4321 о о о & 4321 & 4321 о о о \\
\hline 18 & O O O O O O O & 1 о о о о о о & 21 о о о о о & 321 о о о о & 4321 о о о & 4321 о о о & 54321 о о & 54321 & 54321 o o \\
\hline
\end{tabular}

\begin{tabular}{|c|c|c|c|c|c|c|c|c|c|c|c|c|c|c|c|c|c|c|c|c|c|c|c|c|c|c|c|c|c|c|c|c|c|c|c|c|}
\hline $\log n_{\mathrm{e}}$ & Ly & & & & & & Ly & 12 & & & & & Ly & 13 & & & & & Ly & 14 & & & & & Ly & 15 & & & & & Ly & 16 & & & & \\
\hline 10 & o & o & o & o & o & O O & o & o & o & o & o & O O & o & o & o & o & o & o o & o & $\mathrm{O}$ & o & o & o & O O & o & o & o & o & o & O O & o & o & o & o & o & o o \\
\hline 10.5 & o & o & o & o & o & O o & o & o & o & o & o & O O & o & o & o & o & o & O o & o & O & o & o & o & o o & o & o & o & o & o & O O & o & o & o & o & o & o o \\
\hline 1 & o & o & o & o & $\mathrm{O}$ & o o & $\mathrm{O}$ & o & o & o & o & o o & o & o & o & o & o & o o & o & o & o & o & o & o o & o & o & o & o & o & o o & o & o & o & o & o & O O \\
\hline 1.5 & o & o & o & o & o & o o & o & o & o & o & o & o o & o & o & o & o & o & o o & o & o & o & o & o & o o & o & o & o & o & o & o o & o & o & o & o & o & O o \\
\hline 2 & o & o & o & O & o & O O & o & o & o & o & o & O O & o & o & o & o & o & o o & o & o & o & o & o & O O & o & o & o & o & o & O O & o & o & $\mathrm{o}$ & o & $\mathrm{O}$ & O O \\
\hline 2.5 & o & o & O & $\mathrm{O}$ & $\mathrm{O}$ & O O & $\mathrm{O}$ & o & o & o & o & O O & $\mathrm{O}$ & $\mathrm{O}$ & o & o & o & O o & o & $\mathrm{O}$ & o & o & o & O O & o & o & o & o & o & O O & o & o & o & o & $\mathrm{O}$ & O O \\
\hline 3 & o & o & o & o & o & o o & o & o & o & o & o & o o & o & o & o & o & o & o o & o & o & o & o & o & o o & o & o & o & o & o & o o & o & o & o & o & o & o o \\
\hline 3.5 & o & o & o & o & o & o o & o & o & o & o & o & o o & o & o & o & o & o & o o & o & o & o & o & o & o o & o & o & o & o & o & o o & o & o & o & o & o & o o \\
\hline & o & o & o & o & o & o o & o & o & o & o & o & O O & o & o & o & o & o & o o & o & o & o & o & o & o o & o & o & o & o & o & O O & o & o & o & o & o & O O \\
\hline 14.5 & o & o & o & o & o & o o & o & o & o & o & o & o o & o & o & o & o & o & o o & o & o & o & o & o & o o & 1 & o & o & o & o & o o & 1 & o & o & o & o & O o \\
\hline 15 & o & o & o & o & o & O O & 1 & o & o & o & o & O O & 1 & o & o & o & o & O O & 1 & $\mathrm{O}$ & o & o & o & o o & 1 & o & o & o & o & o o & 2 & 1 & o & $\mathrm{O}$ & o & o o \\
\hline 15.5 & 1 & o & o & $\mathrm{O}$ & o & O O & 2 & 1 & o & o & o & O O & 2 & 1 & o & o & o & O O & 2 & 1 & $\mathrm{O}$ & o & o & O o & 2 & 1 & o & o & o & O O & 2 & 1 & o & o & o & O O \\
\hline 16 & 2 & 1 & o & o & o & o o & 2 & 1 & o & o & o & O O & 3 & 2 & 1 & o & o & O O & 3 & 2 & 1 & o & o & O O & 3 & 2 & 1 & o & o & O O & 3 & 2 & 1 & $\mathrm{O}$ & O & O O \\
\hline 16.5 & 3 & 2 & 1 & o & o & O O & 3 & 2 & 1 & o & o & O O & 3 & 2 & 1 & o & o & O O & 4 & 3 & 2 & 1 & o & o o & 4 & 3 & 2 & 1 & o & o & 4 & 3 & 2 & 1 & o & O O \\
\hline 17 & 4 & 3 & 2 & 1 & o & O o & 4 & 3 & 2 & 1 & o & O O & 4 & 3 & 2 & 1 & o & O O & 5 & 4 & 3 & 2 & 1 & O o & 5 & 4 & 3 & 2 & 1 & O O & 5 & 4 & 3 & 2 & 1 & O O \\
\hline 7.5 & 5 & 4 & 3 & 2 & 1 & o o & 5 & 4 & 3 & 2 & 1 & o o & 5 & 4 & 3 & 2 & 1 & o o & 5 & 4 & 3 & 2 & 1 & o o & 15 & 141 & 131 & 121 & 111 & $10 \mathrm{o}$ & 151 & 14 & 131 & 121 & 111 & $10 \mathrm{o}$ \\
\hline & 15 & 14 & 13 & 12 & 11 & $10 \mathrm{o}$ & 15 & 14 & 131 & 12 & 11 & $10 \mathrm{o}$ & 151 & & 131 & 121 & 111 & $10 \mathrm{o}$ & 15 & & 131 & 121 & & $10 \mathrm{o}$ & & & & & & & & & & & & \\
\hline$T:$ & 2 & 5 & 1 & 2 & 4 & 81 & 2 & 5 & 1 & 2 & 4 & 81 & 2 & 5 & 1 & 2 & 4 & 81 & 2 & 5 & 1 & 2 & 4 & 81 & 2 & 5 & 1 & 2 & 4 & 81 & 2 & 5 & 1 & 2 & 4 & 81 \\
\hline $\log n_{\mathrm{e}}$ & Ly & 17 & & & & & Ly & 18 & & & & & Ly & 19 & & & & & Ly & 20 & & & & & Ly & 21 & & & & & Ly & 22 & & & & \\
\hline & o & o & o & o & o & o o & o & o & o & o & o & o o & o & o & o & o & $\mathrm{O}$ & o o & o & o & o & o & o & o o & o & o & O & o & o & o o & o & o & o & o & $\mathrm{O}$ & o o \\
\hline 0.5 & o & o & o & o & o & o o & o & o & o & o & o & o o & o & o & o & o & o & O o & o & o & o & o & o & o o & o & o & $\mathrm{O}$ & $\mathrm{o}$ & o & o o & o & o & o & o & $\mathrm{o}$ & o o \\
\hline & o & o & o & o & o & o o & o & o & o & o & o & o o & o & o & o & o & o & o o & o & o & o & o & o & o o & o & o & $\mathrm{O}$ & o & o & o o & o & o & o & o & $\mathrm{O}$ & o o \\
\hline 1.5 & o & o & o & o & o & o o & o & o & o & o & o & o o & o & o & o & o & o & o o & o & o & o & o & o & o o & o & o & O & o & o & o o & o & o & o & o & o & O O \\
\hline 2 & o & o & o & o & o & O O & o & o & o & o & o & O O & O & o & o & o & o & O O & $\mathrm{O}$ & O & $\mathrm{O}$ & o & o & O O & o & o & o & o & o & O O & o & o & o & o & o & O O \\
\hline 2.5 & o & o & o & o & o & o o & o & o & o & o & o & o o & o & o & $\mathrm{o}$ & o & o & o o & o & o & o & o & o & o o & o & o & o & o & o & o o & o & o & o & o & o & O o \\
\hline 13 & o & o & O & o & o & O O & O & o & o & o & o & O O & O & o & o & o & o & O O & o & o & o & o & o & O O & o & o & o & o & o & O O & o & o & o & o & o & O O \\
\hline 3.5 & o & o & o & o & o & o o & o & o & o & o & o & o o & o & o & $\mathrm{O}$ & o & o & o o & o & o & o & o & o & o o & o & o & o & o & o & o o & o & o & o & o & o & O O \\
\hline 14 & o & o & o & O & o & O O & $\mathrm{O}$ & o & o & o & o & O O & O & $\mathrm{O}$ & o & o & o & O O & 1 & $\mathrm{O}$ & $\mathrm{O}$ & o & o & O O & 1 & o & o & o & o & O O & 1 & o & o & $\mathrm{O}$ & o & O O \\
\hline 14.5 & 1 & o & o & o & $\mathrm{O}$ & o o & 1 & o & o & o & o & o o & 1 & o & $\mathrm{O}$ & o & o & o o & 1 & o & o & o & o & O o & 2 & 1 & O & o & o & o o & 2 & 1 & o & o & $\mathrm{O}$ & о o \\
\hline 15 & 2 & 1 & o & O & o & O O & 2 & 1 & o & o & o & O O & 2 & 1 & o & o & o & O o & 2 & 1 & o & o & o & O O & 2 & 1 & o & o & o & O O & 3 & 2 & 1 & o & o & O O \\
\hline 15.5 & 3 & 2 & 1 & o & o & O o & 3 & 2 & 1 & o & o & O o & 3 & 2 & 1 & o & o & o c & 3 & 2 & 1 & o & o & o o & 3 & 2 & 1 & o & o & o o & 3 & 2 & 1 & o & o & o o \\
\hline & 3 & 2 & 1 & o & o & o o & 4 & 3 & 2 & 1 & o & o o & 4 & 3 & 2 & 1 & o & o & 4 & 3 & 2 & 1 & o & o & 4 & 3 & 2 & 1 & o & & 4 & 3 & 2 & 1 & o & o o \\
\hline 6.5 & 4 & 3 & 2 & 1 & o & o o & 4 & 3 & 2 & 1 & o & o o & 5 & 4 & 3 & 2 & 1 & o o & 5 & 4 & 3 & 2 & 1 & o o & 5 & 4 & 3 & 2 & 1 & o o & 5 & 4 & 3 & 2 & 1 & o o \\
\hline & 5 & 4 & 3 & 2 & 1 & o o & 5 & 4 & 3 & 2 & 1 & o o & 151 & 141 & 131 & 121 & 111 & $10 \mathrm{o}$ & 15 & & 131 & 121 & 111 & $10 \mathrm{o}$ & 15 & 141 & & & & $10 \mathrm{o}$ & 151 & & 131 & 121 & 111 & $10 \mathrm{o}$ \\
\hline
\end{tabular}

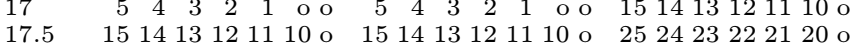
18 
Table 3. continued

\begin{tabular}{|c|c|c|c|c|c|c|c|c|c|c|c|c|c|}
\hline$g n_{\mathrm{e}}$ & $\mathrm{H} \alpha$ & $\mathrm{H} \beta$ & $\mathrm{H} \gamma$ & $\mathrm{H} \delta$ & $\mathrm{H} \epsilon$ & H 8 & H 9 & H 10 & H 11 & & & & \\
\hline & O 00000 & O O O O O O & 000000 & O O O O O O O & O O O O O O O & OOOOOOO & OOOOOOO & O O O O O O O & o & $\mathrm{o}$ & $\mathrm{o}$ & $\mathrm{o}$ & \\
\hline & O O O O O O & O O O O O O & O O O O O O & O O O O O O & O O O O O O & O O OOOOO & O O O O O O O & O O O O O O O & o & o & $\mathrm{O}$ & t & \\
\hline & 000000 & OOOOOOO & O O O O & OOOOOOO & 000000 & OOOOOOOO & 0000 & OOOOOOO & $\mathrm{o}$ & $\mathrm{o}$ & $\mathrm{o}$ & 5 & \\
\hline & OOOOOOO & O O O O O O O & O O O O & O 000000 & O 000000 & O 000000 & OOOOOOO & o o o & $\mathrm{o}$ & $\mathrm{o}$ & $\mathrm{o}$ & 5 & \\
\hline & O O O O O O O & O O O O O O & o o & O O O OO O O & O O O O O O O & OOOOOOO & O OOOOOO & o o o & o & o & o & o & \\
\hline .0 & O O O O O O O & O O O O O O O & o o c & O O O O O O O & O O O OOO O & O O O O O O O & o o o o & o o o & o & o & o & & \\
\hline & o o o & O O OOOOO & o o c & o o c & o o & o o c & o o & O O O & o & b & 0 & 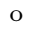 & \\
\hline & o o c & O O O O & o o & o o o & o o & o o c & o o & o o o & o & o & 0 & 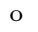 & \\
\hline & o o o & O O O & o o & o o & o o & O O C & o o & o o c & o & o & o & o & \\
\hline 4.5 & o o o & O O O O O O O & o o & o o & & o o c & & o o o & o & o & o & o & \\
\hline & O O C & OOOOOOO & o o & o o & & o o & & & 1 & $\mathrm{o}$ & $\mathrm{o}$ & $\mathrm{o}$ & \\
\hline 5.5 & OOOOOOO & OOOOOOO & o o & o o & o o & o o & & o o & 1 & $\mathrm{o}$ & $\mathrm{o}$ & $\mathrm{o}$ & \\
\hline & OOOOOOO & OOOOOOO & o o & o o o & & & & & 2 & o & $\mathrm{o}$ & $\mathrm{o}$ & \\
\hline 6.5 & O O O O O O O & O O O O O O O & o o & o o o & O O O & o o o & & O o o & 3 & 1 & o & o & \\
\hline & o o c & 1 о о о о о о & 10 & & & & & & 4 & 2 & 1 & o & \\
\hline 1.5 & 10 & 21 & & & & & & & 5 & 3 & 2 & 1 & \\
\hline & 210 & 21 o o & 32 & & & o & & & & & & & \\
\hline & 2512481 & 2512481 & 2512481 & 2512481 & 2512481 & 2512481 & 2512481 & 2512481 & 2 & 1 & 2 & & \\
\hline
\end{tabular}

$\begin{array}{lllllll}\log n_{\mathrm{e}} & \mathrm{Pa} \alpha & \mathrm{Pa} \beta & \mathrm{Pa} \gamma & \mathrm{Pa} \delta & \mathrm{Pa} \epsilon & \mathrm{Pa} 10\end{array}$

\begin{tabular}{|c|c|c|c|c|c|c|c|}
\hline & OOOOOOO & OOOOOOO & OOOOOOO & OOOOOOO & 0000000 & O O O O O O O & 000000 \\
\hline 5 & O O O O O O O & O O O O O O O & O O O O O O O & O O O O O O O & OOOOOOO & O O O O O O O & OOOOOC \\
\hline & OOOOOOO & OOOOOOO & OOOOOOO & 0000000 & OOOOOOO & OOOOOOO & \\
\hline & OOOOOOO & OOOOOOO & OOOOOOO & OOOOOOO & OOOOOOO & OOOOOOO & \\
\hline & OOOOOOO & OOOOOOO & OOOOOOO & OOOOOOO & OOOOOOO & OOOOOOO & \\
\hline 2. & O O O O O O O & O O O O O O O & O O O O O O O & O OOOOOO & O O O O O & O O O O O O O & 80 \\
\hline & O O O O O O O & O O O O O O O & O O O O O O O & O OO OO O O & o o o & o o & o c \\
\hline & O O O O O O O & O OOOOOO & O O O O O O O & O OO OO O O & O O O & O O o o & o o \\
\hline & o o & o o & o o o o & o o & o o & o o & o o \\
\hline & o o & o o & O O O O & o o & o o & o o & \\
\hline & o o & o o & о о о o & o o & o o & o & \\
\hline & o o o & o o & & & & o & \\
\hline & OOOOOOO & o o & & & & o o & \\
\hline & O O O O O O O & o o o & & & & o o & \\
\hline & 1 о о о о о о & 21 о о о о о & 21 o o & 321 & & o o & 4 \\
\hline & & 321 & & 32 & o o & 43 & \\
\hline & 321 & 32 & 432 & 43 & & 54 & \\
\hline & & 2512481 & 2512481 & 2512481 & 2512481 & 2512481 & 25124 \\
\hline
\end{tabular}

\begin{tabular}{|c|c|c|c|c|c|c|c|c|c|c|c|c|c|c|c|}
\hline $\log n_{\mathrm{e}}$ & $\operatorname{Br} \alpha$ & $\operatorname{Br} \beta$ & $\mathrm{Br} \gamma$ & $\operatorname{Br} \delta$ & $\mathrm{Br} \epsilon$ & Br 10 & Br 11 & & & & Br 12 & & & & \\
\hline 10 & O O O O O O O & O O OO O O O & O O O O O O & O O O O O O O & O O O O O O O & O O O O O O O & o & o & o & o o & o & o & o & o & \\
\hline 0.5 & O O O 0000 & 0000000 & 0000000 & 0000000 & 0000000 & OOOOOOO & $\mathrm{o}$ & $\mathrm{o}$ & $\mathrm{o}$ & o o & $\mathrm{o}$ & $\mathrm{o}$ & $\mathrm{o}$ & $\mathrm{o}$ & \\
\hline & O O OOOOO & O OOOOOO & O O O O O O O & OOOOOOO & OOOOOOO & OOOOOOO & $\mathrm{o}$ & $\mathrm{o}$ & $\mathrm{o}$ & o o & $\mathrm{o}$ & $\mathrm{o}$ & $\mathrm{o}$ & $\mathrm{o}$ & \\
\hline 1.5 & O OOOOOO & OOOOOOO & OOOOOOO & OOOOOOO & OOOOOOO & OOOOOOO & $\mathrm{o}$ & $\mathrm{o}$ & o & o o & $\mathrm{o}$ & $\mathrm{o}$ & $\mathrm{o}$ & $\mathrm{o}$ & \\
\hline & O O OOOOO & O O OO OO O & O OO OOO O & O O O O O O O & O O OO OO O & O O O O O O O & $\mathrm{o}$ & o & o & o o & o & o & o & o & \\
\hline 2.5 & O O O O O O O & O O OOOOO & O O O O O O O & O O O O O O O & O O O O O O O & O OOO OO O & $\mathrm{o}$ & o & o & o o & o & o & o & o & \\
\hline 3 & OOOOOOO & OOOOOOO & OOOOOOO & OOOOOOO & OOOOOOO & OOOOOOO & $\mathrm{o}$ & o & o & o o & o & o & o & o & \\
\hline 3.5 & O O O O O O O & O OOOOOO & o o o o c & O O O O O O O & o o & o o o & $\mathrm{o}$ & o & o & o o & o & o & o & o & \\
\hline & OOOOOOO & O O O & o o & o o & o o & o o & $\mathrm{o}$ & o & o & o o & o & o & o & o & \\
\hline 4.5 & OOOOOOO & o o c & o o & o o & o & o o & o & o & o & o o & o & o & o & o & \\
\hline & o o c & o o & o & & & o o & o & o & o & o & 1 & o & o & o & \\
\hline 5.5 & o o & o o & & & & & o & o & o & $\mathrm{o}$ & 2 & o & o & o & \\
\hline & o o & 10 & & & 21 & & 2 & o & o & o & 2 & o & o & o & \\
\hline 6.5 & 10 & & & & & & 3 & 1 & o & o & 3 & 1 & o & o & \\
\hline & 21 & & & & & & 4 & 2 & 1 & $\mathrm{o}$ & 4 & 2 & 1 & o & \\
\hline 7.5 & 321 & & & & & & 54 & 3 & 2 & 1 о о & 5 & 3 & 2 & 1 & \\
\hline & 4321 о о о & 4321 & 4321 о о о & o o & $1 \circ$ o & 321 o o & & & & $1110 \mathrm{o}$ & 15 & & & & \\
\hline
\end{tabular}

